\title{
Complications of Retrievable Inferior Vena Cava Filters: A Retrospective Comparison of Denali and Option-ELITE Filters
}

\author{
Jessica Hightower ${ }^{1}$ Richard Alexander ${ }^{2}$ Evan Lehrman ${ }^{1} \quad$ Ryan Kohlbrenner $^{1} \quad$ Nicholas Fidelman $^{1}$ \\ Andrew Taylor ${ }^{1}$ Kanti Kolli ${ }^{1}$ Antonio Westphalen ${ }^{1}$ Maureen Kohi ${ }^{1}$ \\ ${ }^{1}$ Department of Radiology and Biomedical Imaging, University of \\ California, San Francisco, San Francisco, California, United States \\ 2Department of Internal Medicine, Massachusetts General Hospital, \\ Boston, Massachusetts, United States

\begin{abstract}
Address for correspondence Jessica Hightower, MD, Department of Radiology \& Biomedical Imaging, University of California, San Francisco, 505 Parnassus Avenue, M-391, San Francisco, CA 94143-0628, United States (e-mail: Jessica.Hightower@ucsf.edu).
\end{abstract}

\begin{abstract}
Keywords

- IVC filter

- venous

thromboembolism

- retrievable filters

Purpose To compare the complication rate of the Denali and Option-ELITE inferior vena cava (IVC) filters.

Materials and Methods All patients who had a Denali or Option-ELITE IVC filter placed between March 2014 and March 2016 were retrospectively identified from the electronic medical records. Of the 245 IVC filters placed, the positions of 93 devices (21 Denali and 72 Option-ELITE) were documented on follow-up computed tomography (CT) examinations obtained for reasons unrelated to filter placement. In situations where multiple CT studies were obtained after placement, each study was reviewed, for a total of 200 examinations. Images were assessed for filter complication including caval wall penetration by filter components, associated damage to pericaval tissues, filter tilt, migration, and fracture.

Results Penetration of at least one strut was observed in $13 \%$ of all filters imaged by CT, (Denali: 14\%; Option-ELITE: $13 \%$; $p=1.00$ ). No patients had damage to pericaval tissues or documented symptoms attributed to penetration. Neither the Denali nor the Option-ELITE filters demonstrated significant tilt (>15 degrees of tilt), migration, or fracture. Compared with Denali; the Option-ELITE filter demonstrated an increasing strut penetration rate with longer indwell times $(z=-3.67, p<0.01)$.

Conclusions No significant difference was observed between the rates of caval penetration of the Denali and Option-ELITE IVC filters assessed by CT. Additionally, no findings of filter fracture or migration were noted, suggesting that the Denali filter is non-inferior to the Option-ELITE filter with respect to penetration, fracture, tilt, and migration. The Option-ELITE filter demonstrated a time-dependent tendency toward greater strut penetration with longer indwell times.
\end{abstract}

\section{Introduction}

Inferior vena cava (IVC) filters are routinely placed in patients with venous thromboembolic disease when systemic anticoagulation fails or is contraindicated. Potential complications of retrievable IVC filters include penetration, tilt, migration, and fracture. While the exact clinical significance

received

July 9, 2018

accepted after revision

August 6, 2018

published online

December 10, 2018 of minor penetrance and tilt is not fully understood, filter penetration can be associated with pain, gastrointestinal and retroperitoneal bleeding, organ involvement by penetrating struts, hydronephrosis, aortic pseudoaneurysm, and duodenocaval fistula formation. ${ }^{1}$ The rate of such complications may vary depending on the IVC filter manufacturer. ${ }^{1-4}$ Previously published data from our group demonstrated a
()(1) $\ominus \circledast$
C2018 by Indian Society of Vascular and Interventional Radiology

License terms

10.1055/s-0038-1676196

ISSN 2457-0214. 
$10 \%$ filter penetration rate for the Option filter, the precursor to the Option-ELITE (Argon Medical Devices Inc.). ${ }^{3}$ The Denali filter is a redesign of the Eclipse filter (Bard Peripheral Vascular) that demonstrated low filter complication rates in its original prospective clinical trial; however, follow-up studies have been limited. ${ }^{5}$ The purpose of this study was to characterize the incidence of filter-related complications observed at follow-up computed tomography (CT) for the Denali compared with the Option-ELITE IVC filters.

\section{Materials and Methods}

\section{Patient Population and Inferior Vena Cava Filters}

This Health Insurance Portability and Accountability Act (HIPPA)-compliant retrospective study was approved by our institutional review board, with waiver of informed consent. All consecutive patients who underwent IVC filter placement at our institution between March 2014 and March 2016 were included in the study. Patient demographics are displayed in - Table 1. During this time frame, the Denali and OptionELITE IVC filters were the only two devices used in our institution, and filter choice was based on operator preference.

A total of 245 filters (52 Denali and 193 Option-ELITE) were placed in 239 patients during the study period. Patients with suprarenal and multiple filters were excluded from analysis. An additional inclusion criterion was the availability of at least one CT scan that completely visualized all filter components.

Filter placement was performed via the internal jugular or common femoral approach using standard technique.

\section{Computed Tomography Scans}

Computed tomography images were acquired using General Electric scanners (HiSpeed/LightSpeed, GE Medical Systems). As most follow-up scans were obtained for reasons unrelated to filter placement, there was variability in the protocols, including use of intravenous contrast agent and phase of opacification of the vasculature. Both groups were wellmatched by study type and use of contrast agent ( $\mathbf{- T a b l e} \mathbf{2}$ ). Most studies were CT scans of the abdomen or abdomen/ pelvis (150/200, 75\%), followed by positron emission tomography (PET)/CT scans $(12 / 200,6 \%)$, CT scans of the chest (10/200, 5\%), and CT scans of the lumbar spine or CT myelograms $(10 / 200,5 \%)$. The remaining study types included CT-guided abscess drainages, CT-guided biopsies, CT-guided lumbar punctures, and CT scans obtained for radiation treatment planning. Intravenous contrast agent was used in $66 \%$ (131/200) of studies.

Table 1 Patient demographics

\begin{tabular}{|c|c|c|c|c|}
\hline Variable & Denali & Option & Total & $P$ value \\
\hline Filters & 21 & 72 & 93 & $\mathrm{~N} / \mathrm{A}$ \\
\hline Median age, years (range) & $60(22-76)$ & $60(14-86)$ & $60(14-86)$ & 1.00 \\
\hline Male gender (\%) & $11(52)$ & $38(53)$ & $49(53)$ & 1.00 \\
\hline Placement via internal jugular vein (\%) & $20(95)$ & $60(83)$ & $80(86)$ & 0.28 \\
\hline Placement via common femoral vein (\%) & $1(5)$ & $12(17)$ & $13(14)$ & \\
\hline \multicolumn{5}{|l|}{ Indication for filter placement (in addition to known VTE) } \\
\hline Perioperative prophylaxis, (\%) & $12(57)$ & $36(50)$ & $48(52)$ & 0.63 \\
\hline Known/suspected bleeding risk, (\%) & $8(38)$ & $31(43)$ & $39(42)$ & 0.80 \\
\hline Failed anticoagulation, (\%) & $1(5)$ & $1(1.5)$ & $2(2)$ & 0.40 \\
\hline High embolic risk during initiation of anticoagulation, (\%) & 0 & $3(4)$ & $3(3)$ & 1.00 \\
\hline PE with hemodynamic instability, (\%) & 0 & $1(1.5)$ & $1(1)$ & 1.00 \\
\hline Filters & 21 & 72 & 93 & $\mathrm{~N} / \mathrm{A}$ \\
\hline Median age, years (range) & $60(22-76)$ & $60(14-86)$ & $60(14-86)$ & 1.00 \\
\hline Male gender (\%) & $11(52)$ & $38(53)$ & $49(53)$ & 1.00 \\
\hline Placement via internal jugular vein (\%) & $20(95)$ & $60(83)$ & $80(86)$ & 0.28 \\
\hline Placement via common femoral vein (\%) & $1(5)$ & $12(17)$ & $13(14)$ & \\
\hline \multicolumn{5}{|l|}{ Indication for filter placement (in addition to known VTE) } \\
\hline Perioperative prophylaxis, (\%) & $12(57)$ & $36(50)$ & $48(52)$ & 0.63 \\
\hline Known/suspected bleeding risk, (\%) & $8(38)$ & $31(43)$ & $39(42)$ & 0.80 \\
\hline Failed anticoagulation, (\%) & $1(5)$ & $1(1.5)$ & $2(2)$ & 0.40 \\
\hline High embolic risk during initiation of anticoagulation, (\%) & 0 & $3(4)$ & $3(3)$ & 1.00 \\
\hline PE with hemodynamic instability, (\%) & 0 & $1(1.5)$ & $1(1)$ & 1.00 \\
\hline
\end{tabular}

Abbreviations: N/A, not applicable; PE, pulmonary embolism; VTE, venous thromboembolic disease. 
Table 2 CT data

\begin{tabular}{|c|c|c|c|c|}
\hline Variables & Denali & Option & Total & $P$ value \\
\hline Filters & 21 & 72 & 93 & $\mathrm{~N} / \mathrm{A}$ \\
\hline CT scans & 48 & 152 & 200 & $\mathrm{~N} / \mathrm{A}$ \\
\hline \multicolumn{5}{|l|}{ CT type } \\
\hline Abscess/biopsy/LP, (\%) & $1(2)$ & $5(3)$ & $4(2)$ & \\
\hline Abdomen pelvis, (\%) & $29(60)$ & $108(71)$ & $137(69)$ & \\
\hline Angiography, (\%) & $1(2)$ & $5(3)$ & $6(3)$ & \\
\hline Chest, (\%) & $2(4)$ & $7(5)$ & $9(5)$ & \\
\hline DynaCT, (\%) & $6(13)$ & $7(5)$ & $13(7)$ & \\
\hline Spine (including myelogram), (\%) & $2(4)$ & $8(5)$ & $10(5)$ & \\
\hline Positron emission, (\%) & $4(8)$ & $8(5)$ & $12(6)$ & \\
\hline Radiation treatment planning, (\%) & $3(6)$ & $4(3)$ & $7(3)$ & \\
\hline Contrast use, (\%) & $30(63)$ & $101(66)$ & $131(66)$ & \\
\hline
\end{tabular}

Abbreviations: CT, computed tomography; LP, lumbar puncture; N/A, not applicable.

\section{Imaging Interpretation}

Computed tomography images were reviewed on a picture archiving and communication system (PACS) workstation (Agfa Healthcare) by a single fellowship-trained abdominal imaging attending physician (AW) who was blinded to the filter types being assessed, was unfamiliar with the design features specific to both filters, and was not involved in the placement or removal of the filters. Images were viewed in the axial plane for assessment of penetration, defined as filter strut or anchor measuring $\geq 3 \mathrm{~mm}$ beyond the outer caval wall, noting the number of penetrating struts and involvement of adjacent structures (-Fig. 1). Coronal and sagittal reformations were used to measure tilt, defined as a filter axis $>15$ degrees separated from the longitudinal caval axis. These measurements are in accordance with the Society of Interventional Radiology (SIR) Standards of Practice Committee definitions. ${ }^{3}$ Images were also assessed for fracture of filter components or dislocation, defined in our study as movement cranially beyond the confluence of the renal veins or caudally to the level of the common iliac veins.

\section{Statistical Analysis}

Statistical analysis was performed with SSPS software (version 16.0; SPSS Inc.). Patient demographics and indications for

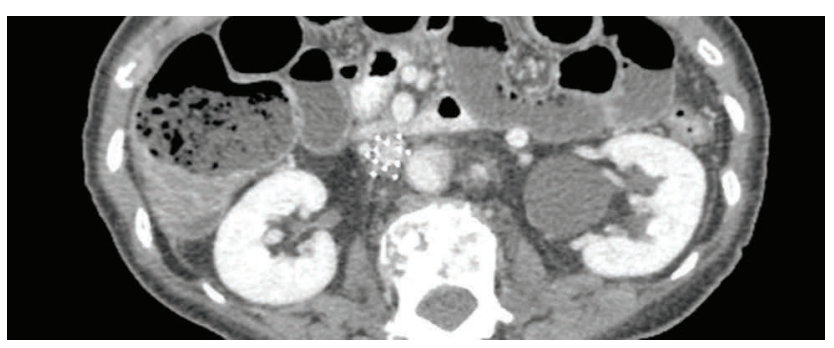

Fig. 1 Axial, contrast-enhanced CT demonstrates penetration of multiple filter struts of $>3 \mathrm{~mm}$ without evidence of injury to adjacent tissues. CT, computed tomography. filter placement were compared between filter types by using a Fisher's exact test for categorical variables and a Student's t-test for continuous variables. The nonparametric MannWhitney test was used to compare follow-up times between filter types, as the distributions were positive-skewed. A Fisher's exact test was used to compare rates of penetration between filter types. To compare rates of penetration over time between the two filter types, linear regression models were used to calculate correlation coefficients, and the Fisher $r$-to- $z$ transformation was applied to the correlation coefficients to determine statistical significance.

\section{Results}

The study population consisted of 93 patients (53\% male, median age: 60 years, range: 14-86) with 21 Denali and 72 Option-ELITE infrarenal IVC filters and at least one follow-up CT examination. A total of 200 CT examinations (48 Denali and 152 Option-ELITE) were available for analysis. The median number of follow-up studies per filter was two for Denali (range: 1-7) and one for Option-ELITE (range: 1-9).

Technical success rate of filter deployment was $100 \%$, and there were no reported immediate complications. Venous access was via the right internal jugular vein in $79 \%$ (73/93) of cases and via the right common femoral vein in 13\% (12/93). Four filters (two Denali and two Option-ELITE) were inserted via the left internal jugular vein and one (Option-ELITE) via the left common femoral vein. There was no statistically significant difference in the insertion via the jugular or common femoral approach between the two devices ( $p=0.28$, - Table 1$)$.

The median time interval between filter insertion and the latest available CT study for all filters was 49 days (mean: 97 days; range: 0-686 days) and was not significantly different between groups (Mann-Whitney $U$ test, $p=0.20$ ). Penetration was observed in 12 of 93 total filters (13\%), with the Denali filters showing a penetration rate of 
$14 \%$ (3/21), compared with $13 \%$ (9/72) in the Option-ELITE group, $(p=1.00)$ (-Table 3 ). Two of the three penetrated Denali filters showed a single strut penetrating on follow-up imaging. The third penetrated Denali filter showed three penetrating struts at 36 days of follow-up, progressing to four penetrating struts at 49 days of follow-up, and remained stable at four penetrating thereafter, observed through 199 total days of follow-up. No filters demonstrated tilt of $>15$ degrees. Neither the Denali nor the Option-ELITE filters demonstrated filter migration or fracture. The Denali filter demonstrated no statistically significant correlation between strut penetration and filter indwell times ( $r$ : 0.065, 95\% confidence interval [CI]: -0.225 to 0.346 ). The Option-ELITE filter did demonstrate a statistically significant correlation between strut penetration and filter indwell times ( $r$ : 0.598, 95\% CI: 0.155-0.443). Using Fisher's $r$-to- $z$ transformation for comparison of the Denali and Option-ELITE filters, this difference was determined to be statistically significant $(z=-3.67, p<0.01)$ ( - Fig. 2).

Table 3 Primary outcomes

\begin{tabular}{|l|l|l|l|l|}
\hline Outcome & Denali & Option & Total & $P$ value \\
\hline $\begin{array}{l}\text { Filters with IVC penetration } \geq 3 \mathrm{~mm} \text { by hook or strut on } \\
\geq 1 \text { study, } \%)\end{array}$ & $3(14)$ & $9(13)$ & $12(13)$ & 1.00 \\
\hline All CT studies with IVC penetration $\geq 3 \mathrm{~mm},(\%)$ & $9(19)$ & $20(13)$ & $29(15)$ & 0.35 \\
\hline Filters with tilt $\geq 15$ degrees on $\geq 1$ study, (\%) & $0(0)$ & $0(0)$ & $0(0)$ & N/A \\
\hline All CT studies with tilted filters $\geq 15$ degrees, (\%) & $0(0)$ & $0(0)$ & $0(0)$ & N/A \\
\hline Fractured filters & 0 & 0 & 0 & N/A \\
\hline Migrated filters & 0 & 0 & 0 & N/A \\
\hline
\end{tabular}

Abbreviations: CT, computed tomography; IVC, inferior vena cava.

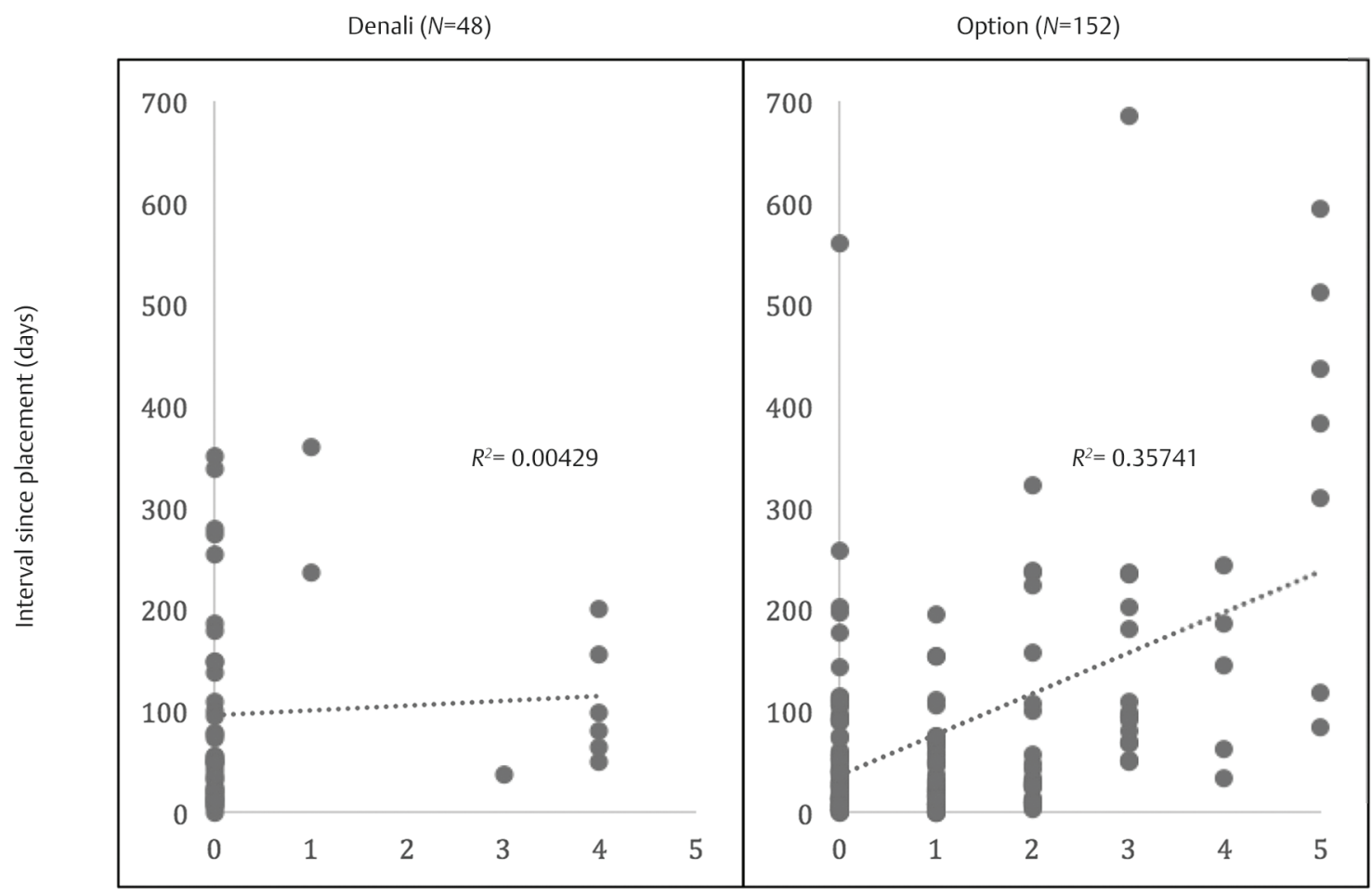

Number of penetrating struts

Fig. 2 Number of penetrating struts over time. There was a time-dependent increase in strut penetration with the Option-ELITE filter, which was not seen with the Denali filter. 


\section{Discussion}

This is the first trial comparing the outcomes of the Denali and Option-ELITE IVC filters. Based on the findings, both filters are associated with a small and similar rate of complication, in particular IVC penetration. However, there is a statistically significant increase in strut penetration with longer indwell times with the Option-ELITE filter when compared with the Denali filter.

The DENALI trial, a prospective, multicenter study, demonstrated no evidence of tilt or fracture of the Denali IVC filter (0/200 and 0/184, respectively), which corresponds to similar absence of these complications on the current study. ${ }^{5}$ However, the DENALI trial demonstrated only a $1.5 \%$ incidence of caval penetration at placement and retrieval, which is markedly lower than the $13 \%$ penetration rate demonstrated in our study. This is likely due to the use of CT for evaluation of strut penetration in our study as opposed to venography used in the DENALI trial. Our results more closely align with those found in the large meta-analysis of IVC filter complications by Jia et al, which demonstrated a 19\% prevalence of overall IVC filter caval penetration. ${ }^{1}$ Tsui et al described comparable outcomes for the Option filter, with low rates of filter tilt and migration (2/323: $0.6 \%$ and 1/323: $0.3 \%$, respectively), but higher rates of strut penetration (57/221: 26\%) compared with the current study. ${ }^{6}$ In both trials, imaging assessment used mixed examination methods-such as plain film, CT, and retrieval cavagram-possibly lending to measurement inaccuracy. ${ }^{7}$

Published data regarding complication rates of the OptionELITE, a variation of the Option device that was redesigned for better retrieval success in January 2014, are limited. In addition to the study by Tsui et al, other previous studies have demonstrated a $10 \%$ rate of strut penetration with the Option filter and no significant tilt or filter fracture, ${ }^{3}$ similar to the $13 \%$ rate of caval penetration and lack of additional filter complications seen with the Option-ELITE filter in our study, suggesting that the Option-ELITE has similar complication rates compared with its precursor. Unlike the Option filter, however, the Option-ELITE did demonstrate a statistically significant increase in time-dependent strut penetration. ${ }^{3}$

There are multiple limitations to the current study. The retrospective nature prohibited the evaluation of complications at defined time points; as such, the wide variability in time intervals between placement and follow-up imaging could result in lead-time bias, where longer time intervals would be more likely to show filter complication than studies performed within shorter time intervals. A single radiologist reviewed all CT examinations for evidence of filter complication, preventing measurement of interobserver variability; the impact of this is considered minimal given the relatively objective use of measurement thresholds to define complications. There was variation in type and coverage of the CT examinations, and many of the CTs did not include the chest. Given the possibility of IVC filter fracture and proximal embolization, the frequency of hardware fracture may be underestimated. The small sample size could create a Type II statistical error where a true difference in complication frequency between filter types is missed due to lack of sufficient power.
There are multiple options for retrievable IVC filters, and the selection of one versus another is often solely dependent on user preference. Knowledge regarding filter complications is helpful in further determining appropriate filter selection. ${ }^{8-13}$ Although the clinical consequences of asymptomatic caval strut penetration is unknown, it seems reasonable that progressive filter penetration could lead to symptomatic penetration over time. The Denali and Option-ELITE IVC filters demonstrate similar rates of strut penetration, migration, and fracture; however, increased strut penetration over time with the Option-ELITE suggests that longer indwelling times may result in greater complication frequency for certain IVC filters. ${ }^{2}$ This underscores the importance of patient follow-up after filter placement to detect complications and to remove the filters as soon as they are no longer clinically indicated. A great deal will be learned about the outcomes of the different available filters following completion of the Predicting the Safety and Effectiveness of Inferior Vena Cava Filters (PRESERVE) trial.

\section{Conflicts of Interest}

No conflicts of interest to disclose.

\section{Acknowledgments}

The authors would like to acknowledge the contribution of our research coordinator, Curt Johanson, to this paper.

\section{References}

1 Jia Z, Wu A, Tam M, Spain J, McKinney JM, Wang W. Caval penetration by inferior vena cava filters: a systematic literature review of clinical significance and management. Circulation 2015;132(10):944-952

2 Mismetti P, Rivron-Guillot K, Quenet S, et al. A prospective long-term study of 220 patients with a retrievable vena cava filter for secondary prevention of venous thromboembolism. Chest 2007;131(1):223-229

3 Olorunsola OG, Kohi MP, Fidelman N, et al. Caval penetration by retrievable inferior vena cava filters: a retrospective comparison of Option and Günther Tulip filters. J Vasc Interv Radiol 2013;24(4):566-571

4 Bos AS, Tullius T, Patel M, et al. Indwelling and retrieval complications of denali and celect infrarenal vena cava filters. J Vasc Interv Radiol 2016;27(7):1021-1026

5 Stavropoulos SW, Chen JX, Sing RF, et al; DENALI Trial Investigators. Analysis of the final DENALI trial data: a prospective, multicenter study of the denali inferior vena cava filter. J Vasc Interv Radiol 2016;27(10):1531-1538.e1

6 Tsui B, An T, Moon E, King R, Wang W. Retrospective review of 516 implantations of option inferior vena cava filters at a single health care system. J Vasc Interv Radiol 2016;27(3):345-353

7 Oh JC, Trerotola SO, Dagli M, et al. Removal of retrievable inferior vena cava filters with computed tomography findings indicating tenting or penetration of the inferior vena cava wall. J Vasc Interv Radiol 2011;22(1):70-74

8 Wang W, Spain J, Tam MD. Acute abdominal pain after retrievable inferior vena cava filter insertion: case report of caval perforation by an option filter. Cardiovasc Intervent Radiol 2011;34(4):883-885

9 Kuo WT, Robertson SW. Bard Denali inferior vena cava filter fracture and embolization resulting in cardiac tamponade: a device failure analysis. J Vasc Interv Radiol 2015;26(1):111-5.e1

10 KuoWT, RobertsonSW, OdegaardJI, Hofmann LV.Complex retrieval of fractured, embedded, and penetrating inferior vena cava filters: 
a prospective study with histologic and electron microscopic analysis. J Vasc Interv Radiol 2013;24(5):622-630.e1, quiz 631

11 Tse G, Cleveland T, Goode S. Ten-year experience of retrievable inferior vena cava filters in a tertiary referral center. Diagn Interv Radiol 2017;23(2):144-149

12 Al-Hakim R, Kee ST, Olinger K, Lee EW, Moriarty JM, McWilliams JP. Inferior vena cava filter retrieval: effectiveness and complications of routine and advanced techniques. J Vasc Interv Radiol 2014;25(6):933-939, quiz 940

13 Angel LF, Tapson V, Galgon RE, Restrepo MI, Kaufman J. Systematic review of the use of retrievable inferior vena cava filters. J Vasc Interv Radiol 2011;22(11):1522-1530.e3 